\title{
Dietary Nucleotides Enhance Plasma Lecithin Cholesterol Acyl Transferase Activity and Apolipoprotein A-IV Concentration in Preterm Newborn Infants
}

\author{
A. SÁNCHEZ-POZO, M. RAMÍREZ, A. GIL, J. MALDONADO, J. P. VAN BIERVLIET, AND \\ M. ROSSENEU
}

Department of Biochemistry and Molecular Biology [A.S.-P, M.R., A.G.] and Department of Pediatrics [J.M.] of the University of Granada, Spain; and Department of Pediatrics [J.P.V.B.] and Department of

Clinical Biochemistry [M.R.], A. Z. Sint-Jan, Brugge, Belgium

\section{ABSTRACT}

The activity of lecithin cholesterol acyl transferase (LCAT), a key enzyme in lipoprotein metabolism, is low in newborn preterm infants. It has been suggested that a normal gastrointestinal function might be necessary to induce a postnatal increase of LCAT activity because apoproteins A-I and A-IV (apoA-I and apoA-IV) synthesized in considerable amounts in the intestine are known activators of LCAT. Dietary nucleotides have been reported to enhance intestinal growth and maturation; therefore, we hypothesized that nucleotide supplementation to formulas for preterm infants may influence LCAT activity. To investigate this hypothesis, two groups of preterm infants were fed either a nucleotide-free formula or a nucleotide-supplemented formula during the first month of life. The plasma LCAT activity, plasma levels of apoA-I and apoA-IV, plasma cholesteryl esters, and plasma fatty acid composition of cholesteryl esters and phospholipids were then determined. Infants receiving nucleotides had higher LCAT activities and apoA-IV levels than those receiving the nucleotide-free formula for a few weeks. The changes in
apoA-IV levels were highly correlated with those of the LCAT activities. However, there were no significant correlations between changes in LCAT activity and plasma cholesteryl esters or phospholipids. These findings indicate that nucleotide supplementation to formulas for preterm infants may improve dietary lipid tolerance by enhancing plasma LCAT activity, probably as a result of an increase in apoA-IV plasma concentrations; they also suggest that nucleotides may enhance apoA-IV synthesis in the intestine during the neonatal period. (Pediatr Res 37: 328333, 1995)
LCAT, lecithir cholesterol acyl transferase
apoA-I, apolipoprotein A-I
apoA-IV, apolipoprotein A-IV
apoB, apolipoprotein B
NFF, nucleotide-free formula
NSF, nucleotide-supplemented formula

LCAT is a plasma enzyme of hepatic origin (1) that catalyses the transfer of an acyl group from lecithin to the 3-hydroxyl group of cholesterol, inducing the formation of cholesteryl ester (2). LCAT is a key enzyme in the metabolism of plasma lipoprotein phospholipids and cholesterol, and it may also indirectly influence the metabolism of triglycerides (3), because an increase in the free cholesterol content of LDL decreases the lipolysis of these particles (4). In addition, LCAT might play an important role in membrane integrity (2).

The LCAT activity is very low in neonates compared with adults; however, a rapid increase takes place immediately after birth (5-7). LCAT activity has been reported to be lower in

Received March 31, 1994; accepted October 10, 1994.

Correspondence and reprint requests: A. Sánchez-Pozo, Department of Biochemistry and Molecular Biology, Faculty of Pharmacy, University of Granada, Campus Cartuja 18071, Granada, Spain.

Supported by grants from the Junta de Andalucia and Puleva, Spain. preterm than in full-term infants $(8-11)$, and this might account for the hyperphospholipidemia and hypercholesterolemia observed during infusion of lipid emulsions (12). The origin of the low LCAT activity is unknown. LCAT activity remains unchanged in preterm infants fed only parenterally (8), suggesting that normal gastrointestinal function might be necessary to induce a postnatal increase of LCAT activity. HDL, which represents LCAT substrate, is synthesized in the intestine in substantial amounts, and apoA-IV, whose cofactor activity is comparable to that of apoA-I in humans (13), is exclusively of intestinal origin $(14,15)$.

Several lines of evidence suggest that nucleotides, given orally, promote intestinal maturation (16-18), thereby increasing plasma lipoprotein concentration, mainly that of $\operatorname{HDL}(19$, 20 ). We recently reported that nucleotide supplementation of milk formulas increases plasma lipoproteins and esterified cholesterol in preterm infants, but it does not affect total 
cholesterol concentration, and we proposed that nucleotides might influence LCAT activity (21). The present report investigates the plasma LCAT activity and the concentrations of the major LCAT activators, i.e. apoproteins A-I and A-IV, together with the fatty acid composition of plasma cholesteryl esters and phospholipids, in preterm infants fed either nucleotide-free or nucleotide-supplemented formula.

\section{METHODS}

\section{Subjects and Protocol}

Two groups of preterm infants with gestational ages of 29-35 wk and birth weights of $1800-2500 \mathrm{~g}$ were studied. Gestational age was determined by the last menstrual period and confirmed by the Dubowitz examination of the newborn (22). Group I consisted of eight preterm newborns fed a standard formula essentially free of nucleotides. The formula for low-birth-weight infants met the European Society for Pediatric Gastroenterology and Nutrition criteria (23). Group II consisted of nine preterm newborns fed the same formula supplemented with nucleotides. A group of five full-term newborns fed human milk was also studied for comparison. The nucleotide content of the supplemented formula, expressed as milligrams per $100 \mathrm{kcal}$, was (after dilution) as follows: uridine monophosphate 0.66 , GMP 0.28 , AMP 0.25 , cytosine monophosphate 0.21 , and inosine monophosphate 0.09 . Only traces of nucleotides were present in the nucleotide-free formula when analyzed by HPLC. The orotate content of the formulas was less than $1.5 \mathrm{mg} / 100 \mathrm{kcal}$. The experimental protocol was approved by the Committee for Research on Human Subjects of the Granada University Hospital, and informed consent was obtained from the parents.

Blood samples were taken from the umbilical cord at birth and from the antecubital vein at wk 1 and 4. Plasma was obtained by centrifugation of the blood collected in EDTA (1 $\mathrm{mg} / \mathrm{mL}$ ) and stored at $-80^{\circ} \mathrm{C}$ until assayed.

\section{Measurements}

Nucleotide analysis. Nucleotides in the formulas were analyzed by reverse-phase HPLC as described previously (24).

Preparation and characterization of apoprotein-lipid complexes for LCAT assay. Complexes were prepared by following the cholate method of Matz and Jonas (25), with a few modifications. Synthetic dipalmitoylphosphatidylcholine and cholesterol were obtained from Sigma Chemical Co. (St. Louis, MO). ApoA-I was prepared from human plasma HDL by following standard procedures (26). Phospholipid/ cholesterol/apoprotein at a weight ratio of 3:0.15:1 was incubated for $16 \mathrm{~h}$ at $43^{\circ} \mathrm{C}$ in the presence of cholate at a phospholipid/cholate weight/weight ratio of 1.7. The cholate was removed by incubation for $3 \mathrm{~h}$ with Bio beads SM2, followed by the recovery of the complexes after centrifugation. The complexes were isolated by gel chromatography on a Superose 6HR column (Pharmacia, Inc., Piscataway, NJ) in a fast protein liquid chromatography system (Waters Instrument Co., Milford, MA).
The chemical composition of the complexes was determined as follows: phospholipids and cholesterol were measured by standard enzymatic colorimetric reactions (Biomerieux), and the apoprotein was measured by analyzing the phenylalanine content by HPLC (27).

Determination of plasma LCAT activity. The enzymatic activity was determined using dipalmitoylphosphatidylcholine/ cholesterol/apoA-I complexes as substrate by quantitating the cholesterol palmitate by HPLC (28). The assay mixture consisted of the phospholipid/cholesterol/apoprotein at a cholesterol concentration of $20 \mu \mathrm{M}, 120 \mu \mathrm{M}$ defatted BSA, $6 \mathrm{mM}$ $\beta$-mercaptoethanol, and $10 \mathrm{mM}$ Tris buffer ( $\mathrm{pH} \mathrm{8)}$ containing $0.15 \mathrm{M} \mathrm{NaCl}, 1 \mathrm{mM} \mathrm{NaN}$, and $0.01 \%$ EDTA in a reaction volume of $700 \mu \mathrm{L}$. After a preincubation period of $20 \mathrm{~min}$ at $37^{\circ} \mathrm{C}$, the enzymatic reaction was initiated by adding $15 \mu \mathrm{L}$ of plasma. The reaction was stopped after $1 \mathrm{~h}$ at $37^{\circ} \mathrm{C}$ by extraction of $625 \mu \mathrm{L}$ of the incubation mixture with $5 \mathrm{~mL}$ of hexane/isopropanol (volume to volume ratio 3:2) containing cholesteryl heptadecanoate (Sigma Chemical Co.) as an internal standard. The tubes were vortexed and centrifuged at 3000 rpm for $10 \mathrm{~min}$, and the supernatant was dried under vacuum, washed twice with chloroform, and dissolved in acetonitrile/ isopropanol for HPLC analysis of the cholesteryl esters formed (28). For each sample, a blank was measured containing all reagents except for the substrate.

The LCAT activity was calculated by subtracting the concentration of cholesteryl palmitate present in the sample blank from that of the sample. The optimal volume of plasma for the assay was determined by using amounts between 15 and $50 \mu \mathrm{L}$ of plasma.

Determination of plasma free and esterified cholesterol. Plasma total and free cholesterol were measured by a combination of an enzymatic method and HPLC (29). Aliquots of 5 $\mu \mathrm{L}$ of plasma were diluted 20 -fold in a $0.05 \mathrm{M}$ Tris buffer $(\mathrm{pH}$ 7.4) containing $0.05 \mathrm{M} \mathrm{MgCl}_{2}, 1 \mathrm{mM}$ DTT, and $0.1 \%$ Triton $\mathrm{X}-100$. One aliquot was used for total cholesterol determination and the other for free cholesterol assay. Esterified cholesterol was calculated as the difference between total and free cholesterol.

Determination of plasma apoproteins. ApoA-I and apoB were measured by a turbidimetric assay using polyclonal monospecific antibodies. Three $\mu \mathrm{L}$ of plasma were incubated for $5 \mathrm{~min}$ with $375 \mu \mathrm{L}$ of diluted antisera in $50 \mathrm{mM}$ Tris buffer (pH 7.3) containing $0.05 \%$ EDTA, $0.9 \% \mathrm{NaCl}, 2 \%$ Tween-20, $0.05 \%$ Kathon C6, and $5 \%$ polyethylene glycol 8000 . Turbidity was measured at $340 \mathrm{~nm}$ in a Technicon RA-1000 autoanalyzer. ApoA-IV was measured by a sandwich-type ELISA (30). The microtiter plate was coated with affinity-purified polyclonal antibodies, then incubated with the sample; the immobilized apoA-IV was detected with a conjugate, prepared with the same polyclonal affinity-purified antibodies, coupled to peroxidase. Calibration curves with purified apoproteins were used for the determination of the plasma concentrations.

Plasma cholesteryl esters and phospholipid fatty acid composition. Plasma lipids were extracted according to the procedure of Haan et al. (31). Cholesteryl esters and phospholipids were separated by thin-layer chromatography on G-60 silica gel plates using a mixture of hexane, ether, and acetic acid 
(80:20:1) according to the procedure of Skipski and Barclay (32). The fractions were extracted with a mixture of chloroform and methanol $(2: 1)$ containing $25 \mathrm{mg} / \mathrm{L}$ of tertbutylhydroxytoluene and were dried under nitrogen. Methyl esters were obtained by the method of Morrison and Smith (33) and analyzed in a Hewlett Packard 5890A gas chromatograph (Palo Alto, CA) using a $30-\mathrm{m} \times 0.7-\mathrm{mm}$ wide-bore capillary SP-2330 column (Supelco, Inc., Bellefonte, PA).

\section{Data Analysis}

Unbalanced repeated-measures analysis of variance was performed to assess the effects of diet and age, and their interaction on LCAT activity, apoprotein levels, cholesterol levels, and fatty-acid composition in cholesteryl esters and phospholipids. No a priori definition of sample size was made. Wald's $\chi^{2}$ statistics were used to analyze the primary effects, and multiple Bonferroni post hoc comparisons were used to evaluate significant effects. A stepwise multiple-regression procedure was used to determine the factors affecting LCAT activity. All tests were performed using the PC-90 version of BMDP program (BMDP Statistical Software, Inc.).

\section{RESULTS}

LCAT activity and apoprotein changes during the neonatal period. The LCAT activities measured in the two groups of preterm infants during the first month of life are presented in Table 1. The enzymatic activity was comparable in the two groups at birth and increased afterward. There was no significant correlation between gestational age and birth weight, and LCAT activity at birth $\left(r^{2}=0.23 ; p=0.198\right)$. The NSF group increased more rapidly than did the NFF group, the difference being significant at 1 wk of life. Compared with the full-term breast-fed infants, preterm infants had significantly lower LCAT activities at birth. After $1 \mathrm{wk}$, the NSF group showed a value close to that of the full-term infants, whereas the NFF group showed significantly lower activity. At 1 mo, all groups showed similar LCAT activities.

The changes in apoprotein concentrations during the first month of life are presented in Table 2. In addition to apoA-I and A-IV, the major activators of human LCAT, we also measured apoB concentration by immunoturbidimetry. All apoprotein concentrations were comparable at birth in the two groups of preterm infants, but the postnatal changes were different. ApoA-I increased from birth to $1 \mathrm{wk}$ and remained constant at 4 wk. No significant differences were observed

Table 1. Plasma LCAT activity in newborn infants

\begin{tabular}{lccc}
\hline \multicolumn{1}{c}{ Age } & $\begin{array}{c}\text { Full-term } \\
(n=5)\end{array}$ & $\begin{array}{c}\text { Preterm NFF } \\
(n=8)\end{array}$ & $\begin{array}{c}\text { Preterm NSF } \\
(n=9)\end{array}$ \\
\hline Cord plasma & $76.6 \pm 9.4$ & $41.0 \pm 4.9^{*}$ & $43.7 \pm 6.9^{*}$ \\
$1 \mathrm{wk}$ & $138.8 \pm 9.2 \dagger$ & $92.8 \pm 8.2^{*} \dagger$ & $179.3 \pm 7.1^{*} \dagger \neq$ \\
$4 \mathrm{wk}$ & $147.3 \pm 9.1 \dagger$ & $131.8 \pm 8.8 \dagger \S$ & $161.5 \pm 16.2 \dagger$ \\
\hline
\end{tabular}

Plasma LCAT activity given in $\mathrm{nmol} / \mathrm{h} / \mathrm{mL}$. Data are expressed as mean \pm SEM. Significant differences are indicated by footnote symbols.

${ }^{*} p<0.05 v s$ full-term infants.

$\dagger p<0.05 v s$ cord plasma.

$\ddagger p<0.01$ vs NFF.

$\S p<0.05$ vs $1 \mathrm{wk}$.
Table 2. Plasma apolipoprotein concentrations in newborn infants

\begin{tabular}{lllll}
\hline Apoprotein & \multicolumn{1}{c}{ Age } & \multicolumn{1}{c}{$\begin{array}{c}\text { Full term } \\
(n=5)\end{array}$} & $\begin{array}{c}\text { Preterm NFF } \\
(n=8)\end{array}$ & \multicolumn{1}{c}{$\begin{array}{c}\text { Preterm NSF } \\
(n=9)\end{array}$} \\
\hline ApoA-I & Cord plasma & $0.79 \pm 0.10$ & $0.48 \pm 0.05^{*}$ & $0.56 \pm 0.04^{*}$ \\
& 1 wk & $0.97 \pm 0.11$ & $0.98 \pm 0.02 \dagger$ & $1.18 \pm 0.08 \dagger$ \\
& 4 wk & $1.30 \pm 0.11 \dagger$ & $1.09 \pm 0.10 \ddagger$ & $1.08 \pm 0.06 \S$ \\
ApoA-IV & Cord plesma & $0.05 \pm 0.02$ & $0.07 \pm 0.01$ & $0.05 \pm 0.01$ \\
& 1 wk & $0.15 \pm 0.05 \dagger$ & $0.11 \pm 0.02 \S$ & $0.25 \pm 0.01 \|^{* *} \dagger \dagger$ \\
& 4 wk & $0.18 \pm 0.09 \S$ & $0.19 \pm 0.01 \ddagger \|$ & $0.20 \pm 0.10 \neq \|$ \\
ApoB & Cord plasma & $0.27 \pm 0.03$ & $0.23 \pm 0.02$ & $0.27 \pm 0.03$ \\
& 1 wk & $0.62 \pm 0.08 \|$ & $0.44 \pm 0.02 \dagger$ & $0.58 \pm 0.06 \| \ddagger \ddagger$ \\
& 4 wk & $0.50 \pm 0.09 \|$ & $0.33 \pm 0.02$ & $0.42 \pm 0.04$ \\
\hline
\end{tabular}

Plasma apolipoprotein concentrations given in $\mathrm{g} / \mathrm{L}$. Data expressed as mean \pm SEM. Significant differences are indicated by footnote symbols.

${ }^{*} p<0.05 v s$ full-term infants.

$\dagger p<0.05$ vs cord plasma.

$\ddagger p<0.01$ vs 1 wk.

$\S p<0.05$ vs 1 wk.

$\| p<0.01 v s$ cord plasma.

${ }^{* *} p<0.01 v s$ full-term infants.

$\dagger+p<0.01 v s \mathrm{NFF}$.

$\ddagger \ddagger p<0.05$ vs NFF.

between the groups. ApoA-IV also increased in both groups but become significantly higher at $1 \mathrm{wk}$ in the NSF compared with the NFF group. The evolution of the apoB concentration was similar to that of apoA-IV. Compared with full-term infants, preterm infants at birth had lower apoA-I levels but reached similar values at $1 \mathrm{wk}$ of life.

Considering postnatal age and apoA-I and apoA-IV concentrations as independent variables, significant correlations were observed with LCAT activity $\left(\mathrm{F}_{\text {postnatal age }}=34.01 ; p<0.01\right.$; $\left.\mathrm{F}_{\mathrm{A}-\mathrm{I}}=18.7, p 0.01 ; \mathrm{F}_{\mathrm{A}-\mathrm{IV}}=80.10, p<0.0001\right)$. Nevertheless, when a multiple correlation was performed, apoA-IV accounted for most of the LCAT variability $\left(r^{2}=0.7184 ; p<\right.$ $0.0001)$.

\section{Plasma Lipids}

Plasma concentrations of free and esterified cholesterol were similar in the two groups of preterm infants at birth, and few changes were observed during the first month of life (Table 3 ). The concentration of free cholesterol was significantly higher

Table 3. Plasma free and esterified cholesterol in newborn infants

\begin{tabular}{llclc}
\hline & \multicolumn{1}{c}{ A.ge } & $\begin{array}{c}\text { Full term } \\
(n=5)\end{array}$ & $\begin{array}{c}\text { Preterm NFF } \\
(n=8)\end{array}$ & $\begin{array}{c}\text { Preterm NSF } \\
(n=9)\end{array}$ \\
\hline Free (mmol/L) & Cord plasma & $0.54 \pm 0.07$ & $0.89 \pm 0.09^{*}$ & $0.88 \pm 0.06^{*}$ \\
& $1 \mathrm{wk}$ & $1.20 \pm 0.09 \dagger$ & $0.93 \pm 0.06$ & $1.17 \pm 0.11$ \\
& $4 \mathrm{wk}$ & $1.29 \pm 0.09 \dagger$ & $1.32 \pm 0.02 \dagger \ddagger$ & $1.07 \pm 0.12$ \\
Esterified (g/L) & Cord plasma & $0.33 \pm 0.04$ & $0.51 \pm 0.07$ & $0.67 \pm 0.16$ \\
& $1 \mathrm{wk}$ & $0.67 \pm 0.05 \dagger$ & $0.57 \pm 0.12$ & $0.76 \pm 0.12$ \\
& $4 \mathrm{wk}$ & $0.88 \pm 0.04 \dagger \S$ & $0.71 \pm 0.04$ & $0.64 \pm 0.07^{*}$ \\
Esterified (\%) & Cord plasma & $61 \pm 1$ & $60 \pm 1$ & $65 \pm 4$ \\
& $1 \mathrm{wk}$ & $59 \pm 1$ & $61 \pm 4$ & $63 \pm 2$ \\
& $4 \mathrm{wk}$ & $64 \pm 1$ & $58 \pm 1$ & $61 \pm 2$ \\
\hline
\end{tabular}

Data are expressed as mean \pm SEM. Significant differences are indicated by footnote symbols.

${ }^{*} p<0.05 v s$ full-tterm infants.

$\dagger p<0.01$ vs cord plasma.

$\ddagger p<0.01$ vs 1 wk.

$\S p<0.05$ vs $1 \mathrm{wk}$. 
at 4 wk than at birth in the NFF group, whereas no changes were observed in the NSF group. Esterified cholesterol levels in the NSF group at $4 \mathrm{wk}$ were significantly lower than those of full-term infants. Compared with full-term infants, preterm infants had levels of free cholesterol that were higher than those of term infants at birth. The LCAT activity was not correlated with either free or esterified cholesterol concentrations. Changes in free and esterified cholesterol were correlated with postnatal age $\left(r^{2}=0.5266 ; p<0.01\right)$.

The fatty acid composition of the cholesteryl esters in the three groups of infants is presented in Table 4. The changes were essentially similar in all groups, with only minor differences. Cholesteryl arachidonate decreased from birth to $4 \mathrm{wk}$, and palmitate decreased from birth to $1 \mathrm{wk}$ and remained constant until 4 wk of life. Cholesteryl linoleate increased after birth in all groups without significant differences.

The fatty acid composition of plasma phospholipids in the three groups of infants is presented in Table 5. There were no significant differences between the groups. The arachidonate and palmitate content decreased whereas the linoleate and oleate content increased with age.

\section{DISCUSSION}

In this study, we found that dietary nucleotides enhance the plasma LCAT activity in preterm infants and that this is correlated with a parallel increase of the levels of plasma apoA-IV. Several studies have reported that LCAT is developmentally regulated and, thereafter, is lower in preterm than in full-term infants at birth (9-11). This observation is relevant clinically because it usually results in hyperphospholipidemia in low-birth-weight infants fed parenterally with lipid emulsions to fulfil their energy requirements (12). The LCAT activity increases rapidly after birth in full-term infants (5-7). In addition, our data show that this increase is slower in preterm infants but that the LCAT activity is similar to that of full-term infants at the end of the first month. In a study of preterm infants receiving exclusively parenteral nutrition, Spear et al. (8) reported that the LCAT activity did not change after birth. These data suggest that the increased LCAT activity might be related to an increase of the activators lipoproteins and apoproteins, which are synthesized in the enterocytes during oral feeding. We and others have described how dietary nucleotides increase intestinal maturation under different circumstances (16-18), possibly because of the limited capacity of the intestine for purine synthesis (34). We previously reported that dietary nucleotides increase HDL, the substrate for LCAT (19-21). In this study, we observed that a variation of the apoA-IV levels might account for the increase in LCAT activity. ApoA-IV is an apoprotein of intestinal origin that increases rapidly together with apoA-I during the neonatal period $(14,35)$, although apo A-I increases only to a minor extent. ApoA-I, the major activator of LCAT, does not seem to be correlated with LCAT activity at birth (11); finally, both apoA-I and apoA-IV levels seem to be affected by diet, although apoA-IV is more sensitive to oral feeding than apoA-I $(13,35)$.

The effect of nucleotides was significant at 1 wk of life but not at $4 \mathrm{wk}$. This transient effect agrees with previous studies that have shown that nucleotides are more effective in conditions such as the early neonatal period characterized by a rapid growth $(16,18)$. It is well known that the major changes in the plasma lipoproteins occur during the first 2 wk of life, reaching a plateau that remains or even decreases at $1 \mathrm{mo}(6)$ and slowly increases afterward. These changes are mediated in part by intestinal maturation as described above, and nucleotides are reported to influence intestinal maturation (16-18), probably by supplying preformed precursors for nucleic acid synthesis and benefitting the cell by saving the high energetic costs of their synthesis. Another possible explanation for the lack of significance at $4 \mathrm{wk}$ is the small sample size, which points to the need for further studies. In any case, the transient increase in LCAT mediated by dietary nucleotides may be of interest because it contributes to a better tolerance of dietary lipids early after birth. No correlation was observed between the changes in LCAT activity and the amount of plasma esterified cholesterol. However, higher levels of esterified cholesterol were present at $1 \mathrm{wk}$, and lower levels of free cholesterol were measured at $4 \mathrm{wk}$ in the newborns receiving the nucleotide-

Table 4. Selected fatty acid composition (\%) of plasma cholesteryl esters in newborn infants

\begin{tabular}{|c|c|c|c|c|c|c|c|c|c|}
\hline \multirow[b]{2}{*}{ Fatty acid } & \multicolumn{3}{|c|}{ Full-term infants $(n=5)$} & \multicolumn{3}{|c|}{ Preterm infants NFF $(n=8)$} & \multicolumn{3}{|c|}{ Preterm infants NSF $(n=9)$} \\
\hline & $\begin{array}{c}\text { Cord } \\
\text { plasma }\end{array}$ & $1 \mathrm{wk}$ & 4 wk & $\begin{array}{l}\text { Cord } \\
\text { plasma }\end{array}$ & 1 wk & $4 \mathrm{wk}$ & $\begin{array}{c}\text { Cord } \\
\text { plasma }\end{array}$ & $1 \mathrm{wk}$ & 4 wk \\
\hline $16: 0$ & $24.6 \pm 1.2$ & $16.5 \pm 1.5$ & $18.5 \pm 1.1$ & $21.9 \pm 0.3$ & $15.6 \pm 0.6^{*}$ & $16.1 \pm 0.5 \dagger$ & $21.9 \pm 0.4$ & $16.5 \pm 0.7^{*}$ & $16.7 \pm 0.9^{*}$ \\
\hline $18: \ln -9$ & $29.1 \pm 1.0$ & $31.7 \pm 3.2$ & $30.0 \pm 1.0$ & $36.0 \pm 0.5$ & $38.0 \pm 1.6$ & $37.8 \pm 1.4$ & $34.5 \pm 0.3$ & $34.4 \pm 1.1$ & $29.8 \pm 1.4$ \\
\hline $18: 2 n-6$ & $17.9 \pm 0.9$ & $33.3 \pm 0.8 \ddagger$ & $35.5 \pm 2.0^{*}$ & $15.0 \pm 0.4$ & $30.9 \pm 2.3^{*}$ & $34.1 \pm 0.9^{*}$ & $13.6 \pm 0.1$ & $32.9 \pm 1.5^{*}$ & $38.6 \pm 1.1 \ddagger \S$ \\
\hline $20: 4 n-6$ & $13.1 \pm 0.9$ & $8.2 \pm 3.3$ & $6.8 \pm 1.5^{*}$ & $11.0 \pm 0.3$ & $4.5 \pm 0.5^{*}$ & $4.2 \pm 0.5^{*}$ & $9.4 \pm 0.3$ & $5.0 \pm 0.4^{*}$ & $4.7 \pm 0.5^{*}$ \\
\hline $22: 6 n-3$ & $1.4 \pm 0.1$ & $0.7 \pm 0.4$ & ND & $1.2 \pm 0.1$ & $0.6 \pm 0.2$ & $0.6 \pm 0.1$ & $1.5 \pm 0.2$ & $0.7 \pm 0.2$ & $0.4 \pm 0.2$ \\
\hline SFA & $29.2 \pm 1.5$ & $19.8 \pm 1.6$ & $23.1 \pm 1.2$ & $27.3 \pm 1.3$ & $18.7 \pm 0.8^{*}$ & $19.8 \pm 0.7^{*}$ & $27.1 \pm 1.4$ & $19.9 \pm 0.7^{*}$ & $20.2 \pm 1.1^{*}$ \\
\hline MUFA & $36.7 \pm 1.6$ & $36.0 \pm 3.6$ & $33.6 \pm 1.5$ & $44.5 \pm 1.4$ & $43.6 \pm 2.3$ & $39.2 \pm 1.4$ & $45.8 \pm 2.1$ & $39.1 \pm 1.3 \ddagger$ & $33.9 \pm 1.3$ \\
\hline LCPUFA n-6 & $14.9 \pm 1.1$ & $9.5 \pm 3.3$ & $7.6 \pm 1.6^{*}$ & $13.9 \pm 1.0$ & $5.8 \pm 0.5^{*}$ & $5.7 \pm 0.5^{*}$ & $11.3 \pm 1.0$ & $6.5 \pm 0.5^{*}$ & $5.9 \pm 0.5^{*}$ \\
\hline LCPUFA n-3 & $1.4 \pm 0.1$ & $0.7 \pm 0.4$ & ND & $1.2 \pm 0.1$ & $0.6 \pm 0.2$ & $0.6 \pm 0.1$ & $1.5 \pm 0.2$ & $0.7 \pm 0.2$ & $0.4 \pm 0.2$ \\
\hline
\end{tabular}

Data are expressed as mean \pm SEM. SFA, total saturated fatty acids; MUFA, total monounsaturated fatty acids; LCPUFA, total long-chain polyunsaturated fatty acids; ND, not detectable. Significant differences are indicated by footnote symbols.

${ }^{*} p<0.01$ vs cord plasma.

$\dagger p<0.01$ vs 1 wk.

$\ddagger p<0.05$ vs cord plasma.

$\S p<0.05$ vs 1 wk. 
Table 5. Selected fatty acid composition (\%) of plasma phospholipids of newborn infants

\begin{tabular}{|c|c|c|c|c|c|c|c|c|c|}
\hline \multirow[b]{2}{*}{ Fatty acid } & \multicolumn{3}{|c|}{ Full-term infants $(n=5)$} & \multicolumn{3}{|c|}{ Preterm infants NFF $(n=\varepsilon)$} & \multicolumn{3}{|c|}{ Preterm infants NSF $(n=9)$} \\
\hline & $\begin{array}{c}\text { Cord } \\
\text { plasma }\end{array}$ & $1 \mathrm{wk}$ & $4 \mathrm{wk}$ & $\begin{array}{c}\text { Cord } \\
\text { plasma }\end{array}$ & $1 \mathrm{wk}$ & 4 wk & $\begin{array}{l}\text { Cord } \\
\text { plasma }\end{array}$ & $1 \mathrm{wk}$ & $4 \mathrm{wk}$ \\
\hline $18: \ln -9$ & $16.0 \pm 1.2$ & $20.0 \pm 0.5$ & $18.0 \pm 1.0$ & $15.9 \pm 0.5$ & $21.9 \pm 1.1^{*}$ & $20.3 \pm 1.0^{*}$ & $15.8 \pm 0.5$ & $20.6 \pm 0.7^{*}$ & $19.1 \pm 1.0^{*}$ \\
\hline $18: 2 n-6$ & $8.2 \pm 0.4$ & $16.1 \pm 1.6^{*}$ & $19.3 \pm 1.4^{*}$ & $7.4 \pm 0.3$ & $19.5 \pm 0.9^{*}$ & $19.8 \pm 0.9^{*}$ & $7.4 \pm 0.4$ & $19.4 \pm 0.9^{*}$ & $20.7 \pm 0.6^{*}$ \\
\hline $20: 4 n-6$ & $11.7 \pm 0.6$ & $6.6 \pm 1.5 \dagger$ & $7.8 \pm 0.9 \dagger$ & $12.3 \pm 0.8$ & $5.7 \pm 0.4^{*}$ & $5.2 \pm 0.6^{*}$ & $12.1 \pm 0.7$ & $6.3 \pm 0.4^{*}$ & $5.6 \pm 0.5^{*}$ \\
\hline MUFA & $18.0 \pm 1.5$ & $20.7 \pm 0.7$ & $18.8 \pm 1.1$ & $20.7 \pm 1.0$ & $23.0 \pm 1.2 \dagger$ & $21.0 \pm 1.1$ & $17.2 \pm 0.9$ & $21.3 \pm 0.8$ & $19.4 \pm 1.0$ \\
\hline LCPUFA $n-6$ & $16.4 \pm 0.8$ & $9.3 \pm 1.9 \dagger$ & $11.6 \pm 0.9 \dagger$ & $17.7 \pm 3.4$ & $10.6 \pm 0.9^{*}$ & $9.1 \pm 1.0^{*}$ & $20.0 \pm 1.8$ & $10.5 \pm 0.7^{*}$ & $9.2 \pm 0.8^{*}$ \\
\hline LCPUFA $n-3$ & $3.7 \pm 0.4$ & $1.3 \pm 0.4$ & $2.3 \pm 0.3$ & $3.1 \pm 0.4$ & $1.7 \pm 0.3$ & $1.3 \pm 0.3 \dagger$ & $2.9 \pm 0.1$ & $1.7 \pm 0.2$ & $1.4 \pm 0.2 \dagger$ \\
\hline
\end{tabular}

Data are expressed as mean \pm SEM. Significant differences are indicated by footnote symbols.

${ }^{*} p<0.01 v s$ cord plasma.

$+p<0.05$ vs cord plasma.

supplemented formula than in those receiving the nucleotidefree formula. The percentage of esterified cholesterol was consistently higher in the NSF group compared with the NFF group. These results are consistent with the observed differences in LCAT activity.

Neither the plasma cholesteryl esters distribution nor the fatty acid composition of plasma phospholipids was affected by the changes in LCAT activity. The changes observed in the fatty acid composition of the two classes of lipids reflected basically the fatty-acid composition of the formulas and agree with previous works (36). Thus, the increase in linoleate and oleate could be expected from the percentages of these two fatty acids in the formulas (15 and $38 \%$ ). The palmitate decrease might occur because of the lower activity of this fatty acid as a substrate of LCAT. The decrease in arachidonate might result from a different mechanism: the low content in the formulas $(0.01 \%)$, a higher requirement of this fatty acid by tissues, or simply the relative increase of other fatty acids.

Our data suggest that nucleotide-supplemented formulas may enhance the synthesis of intestinal apoproteins such as apoA-IV and, concomitantly, the activity of LCAT during the neonatal period. It has been reported that orotate feeding in rats inhibits liver very LDL secretion, probably by interfering with apoB synthesis (37). The mechanism of the inhibition by orotate is linked to a depression of adenine nucleotides and, thus, is rapidly reversed by addition of adenine to the diet (38). In our conditions, the nature of the nucleotide mixture given and the low levels of orotate in the formulas compared with those reported to have a slight effect in humans (39) suggest that no inhibitory effect occurred. Finally, in agreement with our hypothesis, it has been reported that a mixture of dietary nucleotides enhance the expression of intestinal proteins (40).

In conclusion, nucleotide supplementation to preterm-infant formulas may be beneficial to facilitate their lipoprotein metabolism early after birth. Studies should be done comparing breast-fed preterm infants and formula-fed preterm infants to investigate further the potential benefits of nucleotide supplementation in formulas.

Acknowledgments. The authors thank the members of the Department of Clinical Biochemistry of the A. Z. Sint-Jan
Hospital of Brugge, Belgium, for their friendly and valuable cooperation. We also thank D. Nesbitt for the preparation of the manuscript.

\section{REFERENCES}

1. Osuga T, Portman OW 1971 Origin and disappearance of plasma lecithin: cholesterol acyltransferase. Am J Physiol 220:73.5-741

2. Glomset JA 1968 The plasma lecithin: cholesterol acyl transferase reaction. J Lipid Res 9:155-167

3. Marcel YL 1982 Lecithin: cholesterol acyltransferase and intravascular cholesterol transport. Adv Lipid Res 19:85-136

4. Chen $\mathrm{CH}$, Albers JJ 1987 Impaired intermediate-density lipoprotein triglyceride hydrolysis in familial lecithin: cholesterol acyl transferase (LCAT) deficiency. Scand J Clin Lab Invest 47:775-783

5. Dobiasova M, Stozicky F, Kopecka J 1984 Lecithin: cholesterol acyl transferase in children in the early neonate period. Biol Neonate 45:165-168

6. Lane CM, McConathy WJ 1986 Changes in the serum lipids and apolipoproteins in the first four weeks of life. Pediatr Res 20:332-337

7. Cisternas JR, Llanos A, Celedon JM, Riquelme R 1979 Plasma lecithin: cholesterol acyltransferase activity in normal full-term newborn infants. Rev Bras de Pesquisas Med Biol 12:9-15

8. Spear ML, Amr S, Hamosh M, Pereira GR, Corcoran LG, Hamosh P 1991 Lecithin: cholesterol acyltransterase (LCAT) activity during lipid infusion in premature infants. J Pediatr Gastroenterol Nutr 13:72-76

9. Jain SK 1985 Prematurity and lecithin-cholesterol acyl transferase deficiency in newborn infants. Pediatr Res 19:58-6)

10. Papadopoulos A, Hamosh M, Chowdhry P, Scanion JW, Hamosh P 1988 Lecithincholesterol acyltransferase in newborn infants: low activity level in preterm infants J Pediatr 113:896-898

11. Amr S, Chodhry P, Hamosh P, Hamosh M 1988 Low levels of apolipoprotein A-I are not contributors to the low lecithin: cholesterol acyl transferase activity in premature newborn infants. Pecliatr Res 24:191-193

12. Untracht SH 1982 Intravascular metabolism of an artificial transporter of triacylglycerols. Alteration of serum lipoproteins resulting from total parenteral nutrition with Intralipid. Biochim Biophys Acta 711:176-192

13. Steinmetz A, Czekelius P, Thiemann E, Motzny S. Kaffarnik H 1988 Changes of apolipoprotein A-IV in the human neonate: evidence for different inductions of apolipoproteins A-IV and A-I in the postpartum period. Atherosclerosis 69:21-27

14. Black DD, Rohwer PL 1989 Intestinal apolipoprotein synthesis in the newborn piglet: effect of lipid absorption. Pediatr Res 25:108A(abstr)

15. Lefevre M, Roheim PS 1984 Metabolism of apolipoprotein A-IV. J Lipid Res 25:1603-1610

16. Uauy $R \mathbf{1 9 8 9}$ Dietary nucleotides and requirements in early life. In: Lebenthal E (ed) Texbook of Gastroenterology and Nutrition in Infancy. Raven Press, New York, pp $265-280$

17. Núñez MC, Ayudartı MV, Morales D, Suárez MD, Gil A 1990 Effect of dietary nucleotides on intestinal repair in rats with experimental chronic diarrhoea. JPEN J Parenter Enteral Nutt 14:598-604

18. Grimble GK 1994 Dietary nucleotides and gut mucosal defence. Gut 1(suppl 46):51

19. Garcia-Molina V, Aguilera JA, Gil A, Sánchez-Pozo A 1991 Plasma lipoproteins in suckling rats: effect of dietary nucleotides. J Clin Nutr Gastroenterol 6:184-191

20. Sánchez-Pozo A, Pitc. ML, Martínez A. Molina JA, Sánchez-Medina F, Gil A 1986 Effects of dietary nucleotides upon lipoprotein pattern of newborn infants. Nutr Res 6:763-771

21. Sánchez-Pozo A, Mcrillas J, Moltó L, Robles R, Gil A 1994 Dietary nucleotides influence lipoprotein metabolism in newborn infants. Pediatr Res 35:112-116

22. Dubowitz LMQ, Dutowitz V, Galdherg C 1970 Clinical assessment of gestational age in the newborn infant. J Pediatr 77:1 
23. ESPGAN Committee on Nutrition 1987 Nutrition and feeding of preterm infants. Acta Paediatr Scand 336(suppl 3):14

24. Stocchi V, Cucchiarini L, Magnani M, Chiarantini L, Palma P, Crescentini G 1985 Simultaneous extraction and reverse-phase high-performance liquid chromatography determination of adenine and pyridine nucleotides in human red blood cells. Anal Biochem 146:118-124

25. Matz CE, Jonas A 1982 Reaction of human lecithin cholesterol acyltransferase with synthetic micellar complexes of apolipoprotein A-I, phosphatidylcholine, and cholesterol. J Biol Chem 257:4535-4540

26. Van Turnout P, Vercaemst R, Lievens MJ, Caster H, Rosseneu M, Assmamm G 1980 Reassembly of human apoA-I and apoA-Il proteins with unilamellar phosphatidylcholine-cholesterol liposomes. Association kinetics and characterization of the complexes. Biochim Biophys Acta 601:509-523

27. Vanloo B, Morrison J, Fidge N, Lorent G, Lins L, Brasseur R, Ruyschaert JM, Baert J, Rosseneu M 1991 Characterization of the discoidal complexes formed between apoAI-CNBr fragments and phosphatidylcholine. J Lipid Res 32:1253-1264

28. Vercaemst R, Rosseneu M, De Craene I, De Backer G, Kornitzer M 1989 LCAT activity measurements by high-performance liquid chromatography. Atherosclerosis 78:245-250

29. Contreras JA, Castro M, Bocos C, Herrera E, Lasunción MA 1992 Combination of an enzymatic method and HPLC for the quantitation of cholesterol in cultured cells. $J$ Lipid Res 33:931-936

30. Rosseneu M, Michiels G, De Keersgieter W, Bury J, De Slijpere JP, Uterman G, Dieplinger H 1988 Human apolipoprotein A-IV quantitation by sandwich enzymelinked immunosorbent assay. Clin Chem 34:739-743
31. Haan GH, Van der Heide S, Wouters BG 1979 Analysis of fatty acids from human lipids by gas chromatography. J Chromatogr 162:262-266

32. Skipski VP, Barclay M 1969 Thin layer chromatography of lipids. In: Lowenstein JM (ed) Methods in Enzymology. Academic Press, New York, pp 530-598

33. Morrison MR, Smith LM 1968 Preparation of fatty acid methyl esters and dimethylacetals from lipids with boron fluoride methanol. J Lipid Res 5:600-607

34. LeLeiko NS, Bronstein AD, Baliga BS, Munro HN 1983 De novo purine nucleotide synthesis in the rat small and large intestine: effect of dietary protein and purines. J Pediatr Gastroenterol Nutr 2:313-319

35. Labeur C, Van Bierlviet JP, Michiels G, Van Huffel X, Rosseneu M 1989 Apolipoprotein A-IV profiles in newborns: influence of age and nutrition. In: Windler $E$ Greten $\mathrm{H}$ (eds) Intestinal Lipid and Lipoprotein Metabolism. Zuckschewerdt Verlag. Munich, pp 132-135

36. Holman RT 1986 Control of polyunsaturated fatty acids in tissue lipids. J Am Coll Nutr 5:183-211

37. Windmueller HG, Levy RI 1967 Total inhibition of hepatic $\beta$-lipoprotein production in the rat by orotic acid. J Biol Chem 242:2246-2254

38. Windmueller HG 1964 An orotic acid-induced, adenine-reversed inhibition of hepatic lipoprotein secretion in the rat. J Biol Chem 239:530-537

39. Robinson JL, Dombrowski DB, Tauss JR, Jones LR 1985 Assessment in humans of hypolipidemia induced by orotic acid. Am J Clin Nutr 41:605-608

40. LeLeiko NS, Martin BA, Walsh MJ, Kazlow P, Rabinowitz S, Sterling K 1987 Tissue-specific gene expression results from a purine- and pyrimidine-free diet and 6-mercaptopurine in the rat small intestine and colon. Gastroenterology 93:10141020 